Vietnam Journal of Mechanics, VAST, Vol.42, No. 2 (2020), pp. 173-188

DOI: https://doi.org/10.15625/0866-7136/14806

\title{
STRUCTURAL PARAMETER IDENTIFICATION OF A BOLTED CONNECTION EMBEDDED WITH A PIEZOELECTRIC INTERFACE
}

\author{
Thanh-Canh Huynh ${ }^{1,2, *}$ \\ ${ }^{1}$ Institute of Research and Development, Duy Tan University, Da Nang, Vietnam \\ ${ }^{2}$ Faculty of Civil Engineering, Duy Tan University, Da Nang, Vietnam \\ *E-mail: huynhthanhcanh@duytan.edu.vn
}

Received: 05 February 2020 / Published online: 23 April 2020

\begin{abstract}
As the impedance-based technique has been commonly accepted as an innovative structural health monitoring tool, structural identification of a piezoelectric-driven system is of significant interest for damage identification and quantification. This study presents a predictive modelling strategy, which combines the finite element (FE) method with a model-updating approach, for estimating the structural parameters of a piezoelectric interface-bolted connection system. Firstly, the basic operating principle of the piezoelectric-based smart interface is introduced. Secondly, a bolted connection is selected as a host structure to conduct real impedance measurement via the smart interface. Thirdly, a numerical FE model corresponding to the experimental model is established by using a FE program, COMSOL Multiphysics. A sensitivity-based model updating algorithm is adopted to fine-tune the FE model. Finally, structural parameters of the FE model are determined as the numerical impedance signatures match with the measured ones at the same high-frequency band with identical patterns. This study is expected to open an alternative approach for determining unknown structural parameters of the piezoelectric interface-bolted joint system in practice.
\end{abstract}

Keywords: bolted connection, structural identification, piezoelectric interface, impedance method, predictive modelling, model-updating.

\section{INTRODUCTION}

The impedance-based technique has been studied by many researchers and emerged as an innovative damage detection tool [1-4]. Many research efforts have been made on employing the technique to structural health monitoring (SHM) system for civil infrastructure [5-8]. Traditionally, the impedance-based SHM method utilizes PZT (Lead Zirconate Titanate) patches, which are directly surface-bonded to a host structure, to measure electromechanical (EM) impedances in high-frequency range. Since the EM impedance responses contain local dynamics of the host structure, structural damage can be diagnosed by observing the variation in measured EM impedance signatures. The

(C) 2020 Vietnam Academy of Science and Technology 
utilization of high frequencies allows the technique to detect minor changes in a structure induced by damage events. Nonetheless, the direct attachment of the PZTs could cause difficulties in determining sensitive frequency bands for a damage detection task; for a given target structure, the effective frequency band is often unknown and traditionally identified by the try-and-error method $[2,9]$. Moreover, in some cases when the host structure is too stiff, the direct attachment method leads to weak EM impedance responses in the frequency band below $100 \mathrm{kHz}$. Experimental results showed that the effective frequency band can be over $120 \mathrm{kHz}$ for a reinforced concrete girder [10], and even over $800 \mathrm{kHz}$ for a tendon-anchorage joint [11]. It is worthy to note that the effective frequency band higher than $100 \mathrm{kHz}$ has limited the applicability of wireless impedance sensing technology, which can offer autonomous and low-cost SHM $[9,12,13]$.

Several researchers have worked on developing embeddable piezoelectric-based sensing devices for enhancing damage sensitivity of the EM impedance, such as smart aggregates for health monitoring of concrete structure [14], smart washers for critical connection monitoring [9], smart coupons for corrosion monitoring [15]. However, to obtain damage-sensitive EM impedance signatures, those piezoelectric devices must be preinstalled in a monitored structure during the construction, causing the challenge when applied to existing structures. The smart interface technique using a mountable device has been recently developed and can be a promising solution to deal with the above issue $[16,17]$. This technique uses an interfacial structure equipped with a piezoelectric sensor (i.e., the mountable PZT interface) to indirectly acquire EM impedance data from a target structure. The smart interface is designed with bonded sections which allow the interface to be easily mounted on and detached from an existing surface. Moreover, the structural and geometrical properties of the PZT interface can be adjusted to create strong resonances (i.e., sensitive frequencies) in the desired frequency band. The smart interface technique can be used to reduce the effective frequency band, typically below $100 \mathrm{kHz}$, to enable wireless impedance measurements $[12,13]$. Recent studies reported the uses of the smart interface concept for tension force monitoring of axially loaded members [18], strand breakage detection $[19,20]$, and enhancing the EM impedance for SHM of a fibre composite plate [21].

As the EM impedance-based technique has been commonly accepted as an innovative SHM tool, structural system identification of a PZT interface-driven system has become an important issue for damage identification and quantitative damage estimation $[5,22]$. The purpose of the structural system identification is to update a numerical model to represent a real structure. Via the system identification process, unknown structural parameters of a structure can be determined. The system identification can be also used to establish a baseline model to further investigate the performance of an in-situ structure. Many model-updating algorithms have been developed for the structural system identification [23-29]. They could be classified into iterative and non-iterative methods. The non-iterative methods use a one-step process to directly estimate the structural parameters while the iterative methods involve a multi-step model-updating process. In practice, the iterative methods are often preferred because they can provide physically meaningful results in most cases. Among various iterative methods, the modal 
sensitivity-based system identification method is regarded as one of the common modelupdating algorithms and has been successfully employed to identify baseline numerical/analytical models of various structures [28-37].

In the past decades, researchers have used the EM impedance-based technique to detect the changes in the preload force of a bolted connection [38-42]. Because the EM impedance correlates with the structural condition of a bolted connection, the change of bolt-preload force could be detected via observing the change in the EM impedance. Also, some authors attempted to identify the contact stiffness and damping of a bolted joint through model-updating [5]. The smart interface technique was also applied for structural condition monitoring of bolted connections $[8,43,44]$. Although the preload force change can be well detected, it is hard to determine the percentage loss of the preload force (i.e., the damage severity) from the EM impedance measurement $[2,3]$. To estimate the preload force, a possible way is to use impedance measurement and modelupdating of the contact parameter, which was reported in [5]. By updating numerical models corresponding to different preload forces (from the experiment), a relationship between the contact stiffness of a bolted joint and the preload force can be established and further used to estimate the preload force with a known value of the contact stiffness.

So far, there has been no study on the structural system identification of the PZT interface- bolted joint system. The objective of this study is to propose a predictive impedance modelling strategy for the system identification of a piezoelectric interfacedriven system. The main idea is to combine finite element (FE) modelling with a modelupdating approach to estimate the unknown the structural parameters of a bolted joint. At first, the basic operating principle of the piezoelectric-based smart interface is introduced. Next, a bolted girder connection is selected as a host structure to conduct real impedance measurement via the smart interface. Then, a FE model corresponding to the experiment is established by using COMSOL Multiphysics. Afterwards, the FE model is fine-tuned by a sensitivity-based model updating algorithm, to reproduce the measured impedance responses of the connection. Structural parameters of the FE model can be determined as the numerical impedance signatures match with the measured ones at the same high-frequency frequency band with identical patterns. Besides, the fine-tuned FE model can generate high-frequency impedance signatures well-agreed with the measured ones.

\section{PIEZOELECTRIC-BASED SMART INTERFACE TECHNIQUE}

The smart interface technique used in this study was first developed in [17]. The main difference between the smart interface technique and the smart washer technique [9] lies at their designs. The smart washer technique uses a washer-type structure equipped with a PZT [9], so the washer must be pre-inserted into a host structure during the construction. Whereas, the smart interface technique uses a mountable structure equipped with a PZT, so the interface can be mounted on the surface of an existing structure.

As sketched in Fig. 1(a), the piezoelectric-based smart interface is a beam-like structure which is surface-attached to a host structure by two outside bonded sections. The 


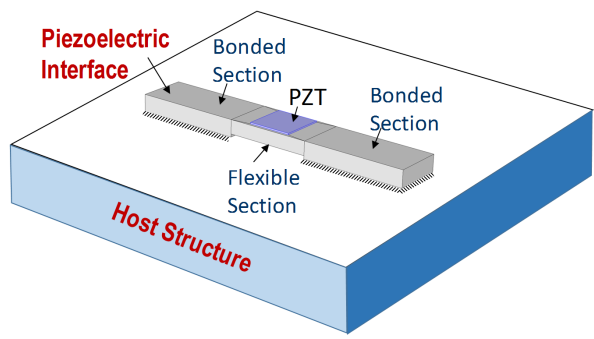

(a) Design of piezoelectric interface

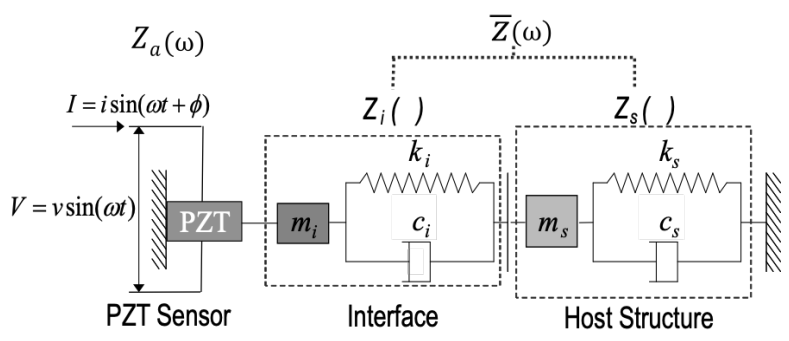

(b) Simplified impedance model

Fig. 1. Piezoelectric-based smart interface technique

middle of the interface, where the PZT (Lead Zirconate Titanate) patch is installed, is designed as a flexible section. The geometrical and material parameters of the flexible section are selected to produce impedance signatures in the desired frequency band. To measure the EM impedance of a host structure, the PZT interface is excited by a harmonic voltage via an impedance analyzer. Under a harmonic excitation, the interface is coupled with the host structure and this coupling is represented in the measured EM impedance. The change in structural parameters of the structure will lead to the alternation of coupling responses, consequently causing the shifts in the measured impedance signatures. So, the health condition of the host structure can be assessed by monitoring EM impedance variations.

Fig. 1(b) shows a simplified impedance model which theoretically describes coupled dynamic responses of the PZT interface-host structure system [22]. The PZT interfacehost structure system is modelled as a 2 degree-of-freedom (dof) spring-mass-damper system, in which $m_{i}, c_{i}, k_{i}$ and $m_{s}, c_{s}, k_{s}$ are the masses, damping coefficients, and spring stiffness of the interface and the host structure. In the model, one dof refers to the host structure represented by the impedance $Z_{s}(\omega)$ and the other refers to the interface represented by its impedance $Z_{i}(\omega)$.

The resultant mechanical impedance of the interface-host structure system $\bar{Z}(\omega)$ at the PZT driving point is defined as the ratio between the excitation force $F_{i}$ and the velocity $x_{i}$, as follows

$$
\bar{Z}(\omega)=\frac{F_{i}}{\dot{x}_{i}}=\frac{K_{11} K_{22}-\left(K_{12}\right)^{2}}{i \omega K_{22}},
$$

in which the terms $\left[K_{i j}\right] ; i, j=1: 2$ are the dynamic stiffness of the 2-dof system, as follows

$$
\left[\begin{array}{ll}
K_{11} & K_{12} \\
K_{12} & K_{22}
\end{array}\right]=\left[\begin{array}{cc}
-\omega^{2} m_{i}+i \omega c_{i}+k_{i} & -i \omega c_{i}-k_{i} \\
-i \omega c_{i}-k_{i} & -\omega^{2} m_{s}+i \omega\left(c_{i}+c_{s}\right)+\left(k_{i}+k_{s}\right)
\end{array}\right] .
$$

The overall EM impedance of the PZT interface-host structure system can be obtained $[1,5]$, as follows

$$
Z(\omega)=\left\{i \omega \frac{w_{a} l_{a}}{t_{a}}\left[\hat{\varepsilon}_{33}^{T}-\frac{1}{Z_{a}(\omega) / \bar{Z}(\omega)+1} d_{31}^{2} \hat{Y}_{11}^{E}\right]\right\}^{-1},
$$


where $\hat{Y}_{11}^{E}=(1+i \eta) Y_{11}^{E}$ is the complex Young's modulus of the PZT patch at zero electric fields; $Y_{11}^{E}(\omega)$ is Young's modulus of the PZT patch; $\hat{\varepsilon}_{33}^{T}=(1-i \delta) \varepsilon_{33}^{T}$ is the complex dielectric constant at zero stress; $\varepsilon_{33}^{T}$ is the dielectric constant of the PZT patch; $d_{31}$ is the piezoelectric coupling constant in 1-direction at zero stress; $w_{a}, l_{a}, t_{a}$ are the width, length, and thickness of the PZT patch, respectively; $i$ is the imaginary unit $\left(i^{2}=-1\right)$; the parameters $\eta$ and $\delta$ are structural damping loss factor and dielectric loss factor of the PZT patch, respectively; $Z_{a}(\omega)$ is the mechanical impedance of the PZT patch [1].

The simplified impedance model should contain two resonant peaks in its EM impedance signatures that represent the two coupled vibration modes of the PZT interface-host structure system. When the host structure is damaged, its structural parameters $m_{s}, c_{s}, k_{s}$ are altered, resulting in the variation in the overall impedance according to Eq. (2) and Eq. (3). By quantifying the impedance changes, the structural damages can be detected.

\section{EXPERIMENTAL INVESTIGATION ON BOLTED CONNECTION}

\subsection{Experimental setup}

Impedance measurement via the smart interface was conducted on a lab-scaled steel beam connection. Fig. 2(a) shows the schematic of the test-setup of the beam. The beam of $3.96 \mathrm{~m}$ was assembled from two H-shaped beams $(\mathrm{H}-200 \mathrm{~mm} \times 180 \mathrm{~mm} \times 8 \mathrm{~mm} \times$ $100 \mathrm{~mm}$ ) by splice plates $(200 \mathrm{~mm} \times 310 \mathrm{~mm} \times 10 \mathrm{~mm})$ and 8 bolts at ever flange (Korean standard bolt, $\phi 20 \mathrm{~mm}$ ). Then the beam was simply supported by four steel rods. The geometric parameters of the splice connection and the PZT interface are displayed in Fig. 2(b). The piezoelectric interface, which was fabricated from an aluminium plate, has a flexible section of $33 \mathrm{~mm} \times 30 \mathrm{~mm} \times 4 \mathrm{~mm}$ and two outside bonded sections of $33 \mathrm{~mm}$ $\times 35 \mathrm{~mm} \times 5 \mathrm{~mm}$. The interface was equipped with a PZT-5A of $15 \mathrm{~mm} \times 15 \mathrm{~mm} \times$ $0.51 \mathrm{~mm}$ at the flexible section.

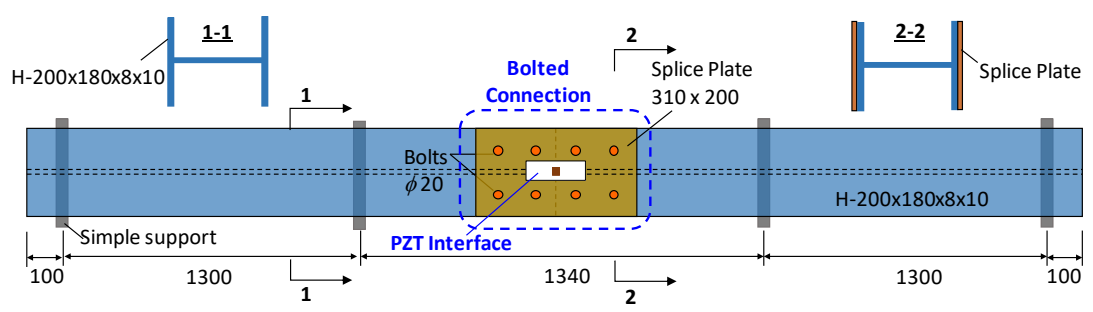

(a) Geometric parameters of the lab-scaled steel beam (mm)

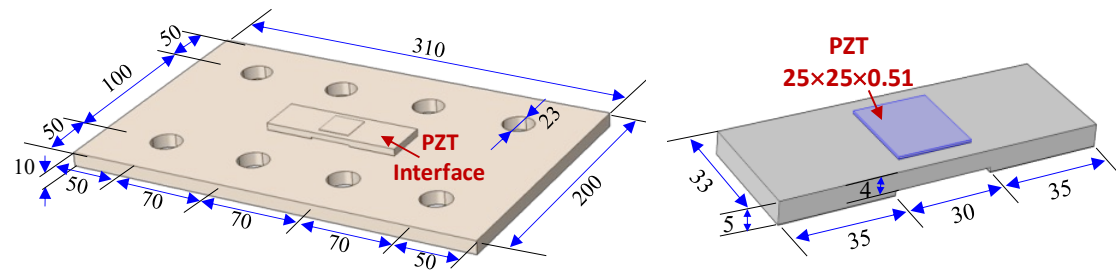

(b) Geometric parameters of the splice connection and the PZT interface (mm) 


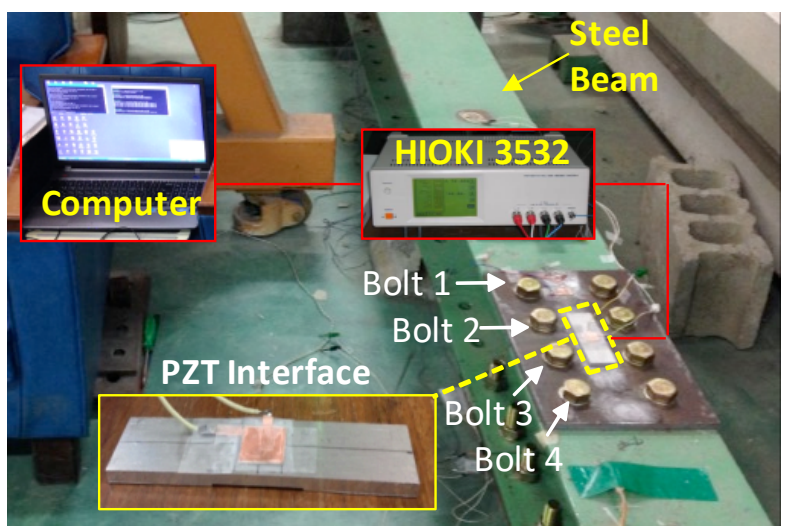

(c) Real view of the experimental model

Fig. 2. Experimental setup of the lab-scaled beam connection

Fig. 2(c) shows the real setup of the lab-scaled bolted connection. All bolts were fastened by a torque of $160 \mathrm{Nm}$. The smart interface was surface-mounted to the splice plate via two instant adhesive layers (Loctite 401). The PZT patch was also bonded to the flexible section via the instant adhesive layer. The impedance measurement system included a computer connected to an impedance analyzer (HIOKI 3532) which was consequently connected to the PZT patch on the smart interface, see Fig. 2(c).

\subsection{Impedance measurement via smart interface}

The EM impedance signatures were obtained from the bolted connection via the smart interface. The impedance analyzer, HIOKI 3532, generated the harmonic excitation and recorded the EM impedance signatures which were finally displayed on a computer. Particularly, the PZT was excited by a $1 \mathrm{~V}$ harmonic voltage in the frequency range of 10-55 kHz. During the impedance measurement, the room temperature was controlled at $21^{\circ} \mathrm{C}$ to avoid any effect caused by temperature changes.

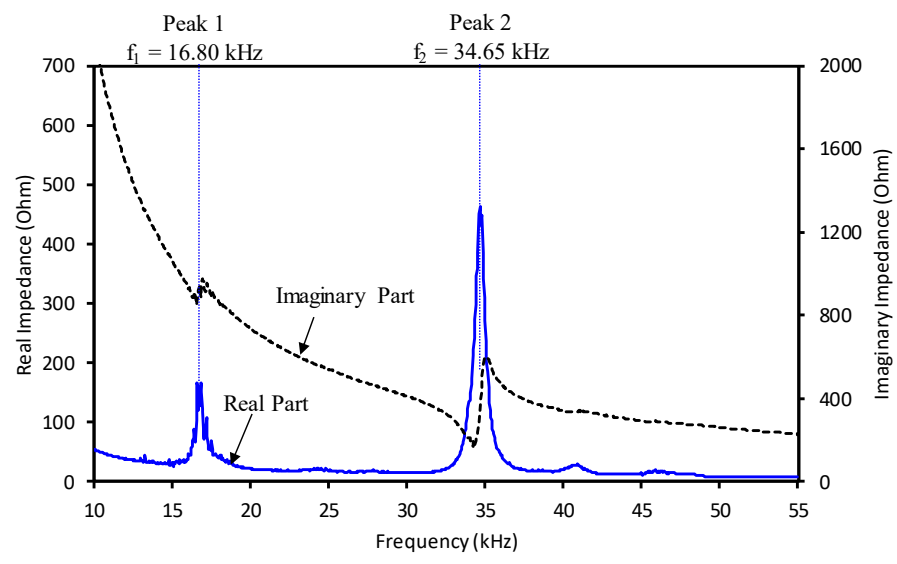

Fig. 3. Experimental EM impedance signatures 
Real and imaginary parts of the measured EM impedance are shown in Fig. 3, respectively. There were two strong resonances of the EM impedance in 15-18 kHz and $33-36 \mathrm{kHz}$, where the aspect of the real part became significant as that of the imaginary part. The first resonance (Peak 1) occurred at the frequency of $16.80 \mathrm{kHz}\left(f_{1, \operatorname{Exp}}\right)$ and the second one (Peak 2) occurred at $34.65 \mathrm{kHz}\left(f_{2, E x p}\right)$. These resonant impedance peaks represented the coupled vibrations of the smart interface-bolted connection system. It is noted that the contribution of the structural impedance to the overall EM impedance was significant at resonance [9]. Thus, the resonant bands of the EM impedance should be employed to maximize the damage detectability.

\section{NUMERICAL MODELLING OF PZT INTERFACE-DRIVEN SYSTEM}

\subsection{Initial finite element model}

Due to its strong modelling capability of the piezoelectric effects, the FE program, COMSOL Multiphysics, was employed to build a numerical model corresponding to the experimental model. The model consisted of the PZT patch and its bonding layer, the interface body and its bonding layers, and the splice plate, as shown in Fig. 4. Dimensional parameters of the model were based on the actual geometry of the experimental model. The FE model was meshed by 3D solid elements. The elastic hexahedral elements were used for the smart interface and the bonding layers, the elastic prism elements were used for the splice plate, and the piezoelectric hexahedral elements were used for the

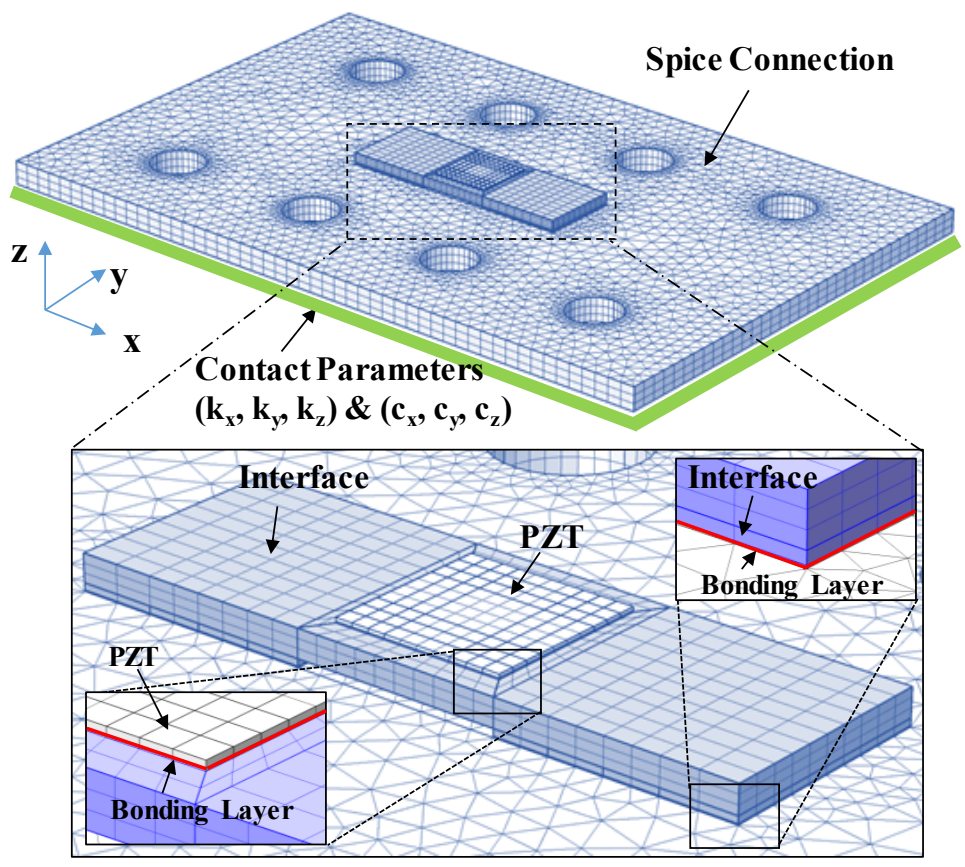

Fig. 4. FE modelling of the experimental model 
PZT patch. To simulate the combined piezoelectric and mechanical effects, the two modules of COMSOL: Piezoelectric Devices and Structural Mechanics were coupled. Similar to the experiment, a harmonic voltage of $1 \mathrm{~V}$ was applied to the top surface of the PZT patch while the bottom was set as the ground electrode. The EM impedance was computed by the ratio between the input voltage and the output current. The contact parameters were used to simulate the interaction between the splice and the remainder of the connection [5,45]. Accordingly, three-dimensional spring system $\left(k_{x}, k_{y}, k_{z}\right)$ and a three-dimensional dashpot system $\left(c_{x}, c_{y}, c_{z}\right)$ were used to support the smart interfaceconnection splice system, see Fig. 4 . It is noted that the value of the contact spring and dashpot represents the amount of the bolt preload introduced into the connection [5].

In the FE model, the splice plate was assigned by the steel material with Young's modulus $E=200 \mathrm{GPa}$, Poisson's ratio $v=0.33$, mass density $\rho=7850 \mathrm{~kg} / \mathrm{m}^{3}$. The interface body was assigned by the aluminum material with $E=70 \mathrm{GPa}, v=0.33$, and $\rho$ $=2700 \mathrm{~kg} / \mathrm{m}^{3}$. The PZT was assigned by the piezoelectric materials with the structural and piezoelectric properties obtained from [44]. The $z$-directional contact stiffness was initially set as $k_{z}=5.0 \times 10^{11} \mathrm{~N} / \mathrm{m}^{2} / \mathrm{m}$ and the $x$-directional and $y$-directional contact stiffness $k_{x}$ and $k_{y}$ were assumed to be $2.5 \times 10^{11}$. The dashpot system was simulated by the damping ratios $\xi_{x}=\xi_{y}=\xi_{z}=0.01$. From previously published data [46, 47], the bonding layers were first assumed to have the thickness $0.2 \mathrm{~mm}$, Young's modulus $3 \mathrm{GPa}$, Poisson's ratio 0.38 , and the mass density $1700 \mathrm{~kg} / \mathrm{m}^{3}$. As compared to the simulation of tendon-anchorage connection in [22], the complexity of the FE model in this study lies at the modelling of thin bonding layers (of the PZT and the smart interface), which yields additional unknown parameters (bond thickness and stiffness) for model-updating.

\subsection{Impedance response of initial FE model}

The EM impedance of the initial FE model was analyzed in the frequency range of 10-55 kHz. The numerical EM signatures obtained from the initial FE model were compared with the ones measured from the experimental test, as shown in Fig. 5. Similar to the experiment, the numerical simulation showed two remarkable resonant peaks in

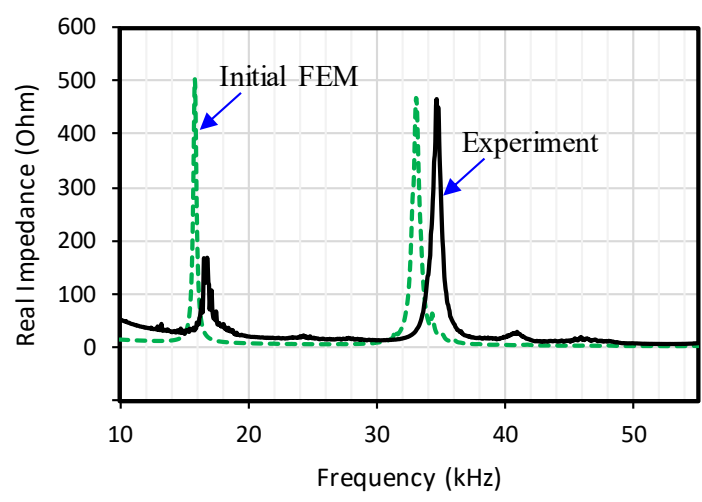

(a) Real impedance

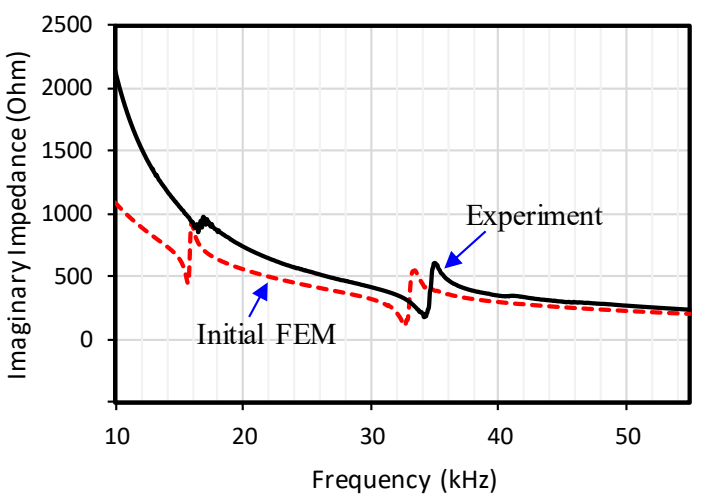

(b) Imaginary impedance

Fig. 5. EM impedance signatures: initial FE model vs experimental model 
the real impedance signature of 10-55 kHz (see Fig. 5(a)). The frequencies of Peak 1 and Peak 2 were found at $15.85 \mathrm{kHz}\left(f_{1, F E M}\right)$ and $33.05 \mathrm{kHz}\left(f_{2, F E M}\right)$, respectively. However, the FE model predicted the frequencies of Peak 1 and Peak 2 different from the experimental model (see Fig. 5(a)). The differences were also found in the imaginary impedance signatures (see Fig. 5(b)). To regenerate the experimental impedance signatures at the same frequency range with identical patterns, the structural parameters of the FE model should be fine-tuned.

\section{STRUCTURAL IDENTIFICATION OF PZT INTERFACE-BOLTED CONNECTION SYSTEM}

\subsection{Model-updating algorithm}

As one of the common model-updating algorithms [28,30-35], the modal sensitivitybased system identification method was adopted to identify the FE model of the previous experimental model. The modal sensitivity-based method can be performed via four steps [36]: (1) selecting the NE model-updating parameters of the FE model and measuring a set of $\mathrm{M}$ modal frequencies from the target structure; (2) computing the modal sensitivity of the selected model-updating parameters of the FE model; (3) estimating the fractional changes in these parameters by the modal sensitivity equation; and (4) repeating the whole process until the differences in the modal frequencies between the FE model and the experimental model are minimal.

Supposing $k_{j}^{*}$ and $k_{j}$ are the $j^{\text {th }}$ model-updating parameter of the target structure and the FE model, respectively. It is noted that the value of $k_{j}^{*}$ is unknown while that of $k_{j}$ is known. The unknown parameter $k_{j}^{*}$ is related to the known parameter $k_{j}$ and its fractional change $\alpha_{j}\left(\alpha_{j} \geq-1\right)$ based on the following expression

$$
k_{j}^{*}=k_{j}\left(1+\alpha_{j}\right) .
$$

The modal sensitivity $\left(S_{i j}\right)$ of the $i^{\text {th }}$ impedance peak $\left(f_{i}\right)$ regarding the $j^{\text {th }}$ structural stiffness parameter $\left(k_{j}\right)$ is computed, as follows

$$
S_{i j}=\frac{\delta f_{i}^{2}}{\delta k_{j}} \frac{k_{j}}{f_{i}^{2}},
$$

where $\delta k_{j}$ is the $k_{j}$ parameter's first order perturbation causing the frequency change in the impedance peak $\delta k_{i}^{2}$.

With a known modal sensitivity matrix [S], the fractional changes in the modelupdating parameters can be given as

$$
\{\boldsymbol{\alpha}\}=[\mathbf{S}]^{-\mathbf{1}}\{\mathbf{Z}\},
$$

where is $\{\boldsymbol{\alpha}\}$ is a vector of NE elements; $\{\mathbf{Z}\}$ is a vector of $M$ elements which contains the fractional difference in the modal frequencies between the FE model and the experimental model; and [S] is a M $\times$ NE matrix. If the [S] matrix is non-square, the inverse of the [S] matrix is approximated by using pseudo-inverse technique. Thus, the fractional 
changes in the model-updating parameters is obtained as

$$
\{\alpha\}=\mathbf{S}^{\mathbf{T}}\left[\mathrm{SS}^{\mathrm{T}}\right]^{-1}\{\mathrm{Z}\}
$$

It is noted that the pseudo-inverse technique provides the least variance solution of the linear system. If the number of the parameters was much less than that of available modal frequencies, the system is ill-conditioned.

A criterion for the convergence between the measured and numerical modal frequencies is defined for the fine-tuning process, as follows

$$
\left|Z_{i}\right|=\left|\frac{f_{i, E x p}^{2}-f_{i, F E M}^{2}}{f_{i, F E M}^{2}}\right| \leq \text { tolerance, }
$$

where $f_{i, E x p}^{2}$ is the $i^{t h}$ peak frequency measured from the experimental model and $f_{i, F E M}^{2}$ is the $i^{\text {th }}$ peak frequency of the FE model.

\subsection{Selection of model-updating parameters}

The selection of proper structural parameters in the FE model is the key to succeed in a model-updating process. Typically, the structural parameters, which are unknown due to the lack of information, should be selected. For well-known materials such as structural steel, aluminium alloy and PZT-5A, no turning process was performed. The standard values of these material properties were used. As compared to those materials, the properties of the bonding layers are relatively uncertain parameters and dependent on installation methods as well as curing conditions during the experiment.

It is found from the previous studies [21,22] that the Poison's ratio of the bonding layer is about 0.38 with a minor deviation and the mass density is stable at $1700 \mathrm{~kg} / \mathrm{m}^{3}$; meanwhile, Young's modulus and the thickness of the bonding layer have a large deviation. Young's modulus of the bonding layer has significant impacts on the high-frequency response of PZT sensors. Moreover, the values of the contact stiffness $k_{x}, k_{y}, k_{z}$ are also unknown and dependent on the fastening force of the bolts. There are no direct formulas that can estimate these contact parameter; the only way is to update them. From the previous study, the values of $k_{x}$ and $k_{y}$ were found to be around half of $k_{z}$ [17] Therefore, to reduce the number of unknown parameters, $k_{x}$ and $k_{y}$ were set as $0.5 k_{z}$. Due to the limited number of available modal frequencies (only two), it is assumed that the PZT bonding layer and the interface-splice bonding layer has the same thickness and the same material properties to secure the possibility of model-updating convergence.

From the above discussions, at least three unknown structural parameters should be selected as the model-updating parameters, including (1) the bond thickness $t_{b},(2)$ the bond Young's modulus $E_{b}$, and (3) the contact stiffness $k_{z}$.

\subsection{Updating finite element model}

The FE model was fine-tuned by the modal sensitivity-based algorithm. The values of updating parameters were constrained to be larger than 0 . The experimental frequencies corresponding to the two impedance peaks were used as the target frequencies for 
model-tuning. The FE model was converged after 27 iterations with the average tolerance of two peaks $<1 \%$. Fig. 6(a) showed the convergence of the two peak frequencies of the FE model. In the beginning, the frequency error between the initial FE model and the experimental model was about $6 \%$ for Peak 1 and about $5 \%$ for Peak 2; however, the error was decreased to $0.3 \%$ for Peak 1 and $1.3 \%$ for Peak 2 at the $27^{\text {th }}$ iteration (the average error of $0.8 \%$ ).

Table 1. Model-updating parameters and peak frequencies during the system identification process

\begin{tabular}{cccccccccccc}
\hline Iter. & $f_{1}(\mathrm{kHz})$ & $f_{2}(\mathrm{kHz})$ & $t_{b}(\mathrm{~mm})$ & $E_{b}(\mathrm{GPa})$ & $k_{z}\left(\mathrm{~N} / \mathrm{m}^{2} / \mathrm{m}\right)$ & Iter. & $f_{1}(\mathrm{kHz})$ & $f_{2}(\mathrm{kHz})$ & $t_{b}(\mathrm{~mm})$ & $E_{b}(\mathrm{GPa})$ & $k_{z}\left(\mathrm{~N} / \mathrm{m}^{2} / \mathrm{m}\right)$ \\
\hline- & 15.85 & 33.05 & 0.2000 & 3.0000 & $3.00 \mathrm{E}+12$ & 14 & 16.48 & 33.79 & 0.1716 & 5.7046 & $5.89 \mathrm{E}+12$ \\
1 & 15.97 & 33.18 & 0.1823 & 3.1701 & $3.01 \mathrm{E}+12$ & 15 & 16.50 & 33.84 & 0.1854 & 6.3356 & $5.86 \mathrm{E}+12$ \\
2 & 16.06 & 33.32 & 0.1739 & 3.4146 & $3.07 \mathrm{E}+12$ & 16 & 16.52 & 33.88 & 0.1969 & 6.9364 & $5.68 \mathrm{E}+12$ \\
3 & 16.13 & 33.35 & 0.1698 & 3.6859 & $3.16 \mathrm{E}+12$ & 17 & 16.61 & 33.98 & 0.1781 & 7.5047 & $7.02 \mathrm{E}+12$ \\
4 & 16.19 & 33.52 & 0.1636 & 3.8197 & $3.38 \mathrm{E}+12$ & 18 & 16.69 & 34.07 & 0.1596 & 8.4948 & $6.09 \mathrm{E}+12$ \\
5 & 16.24 & 33.52 & 0.1583 & 3.9442 & $3.57 \mathrm{E}+12$ & 19 & 16.75 & 34.15 & 0.1415 & 9.5810 & $5.24 \mathrm{E}+12$ \\
6 & 16.28 & 33.71 & 0.1544 & 4.0429 & $3.83 \mathrm{E}+12$ & 20 & 16.73 & 34.13 & 0.1457 & 9.6097 & $5.25 \mathrm{E}+12$ \\
7 & 16.32 & 33.47 & 0.1505 & 4.1442 & $4.02 \mathrm{E}+12$ & 21 & 16.73 & 34.12 & 0.1412 & 9.2944 & $5.28 \mathrm{E}+12$ \\
8 & 16.34 & 33.57 & 0.1488 & 4.1915 & $4.46 \mathrm{E}+12$ & 22 & 16.73 & 34.12 & 0.1368 & 8.9856 & $5.30 \mathrm{E}+12$ \\
9 & 16.37 & 33.61 & 0.1470 & 4.2403 & $4.90 \mathrm{E}+12$ & 23 & 16.74 & 34.10 & 0.1325 & 8.6870 & $5.33 \mathrm{E}+12$ \\
10 & 16.39 & 33.64 & 0.1458 & 4.2763 & $5.38 \mathrm{E}+12$ & 24 & 16.74 & 34.09 & 0.1282 & 8.3784 & $5.35 \mathrm{E}+12$ \\
11 & 16.41 & 33.65 & 0.1448 & 4.3041 & $5.92 \mathrm{E}+12$ & 25 & 16.77 & 34.15 & 0.1050 & 8.5700 & $5.85 \mathrm{E}+12$ \\
12 & 16.42 & 33.66 & 0.1443 & 4.3208 & $6.55 \mathrm{E}+12$ & 26 & 16.83 & 34.17 & 0.0948 & 8.8500 & $6.25 \mathrm{E}+12$ \\
13 & 16.45 & 33.715 & 0.1476 & 4.7575 & $6.02 \mathrm{E}+12$ & 27 & 16.85 & 34.20 & 0.0920 & 9.0200 & $6.72 \mathrm{E}+12$ \\
\hline
\end{tabular}

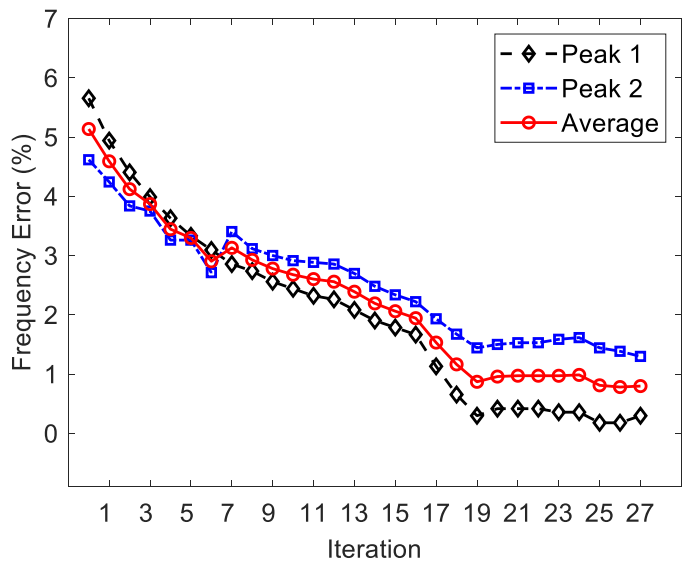

(a) Model-updating frequencies

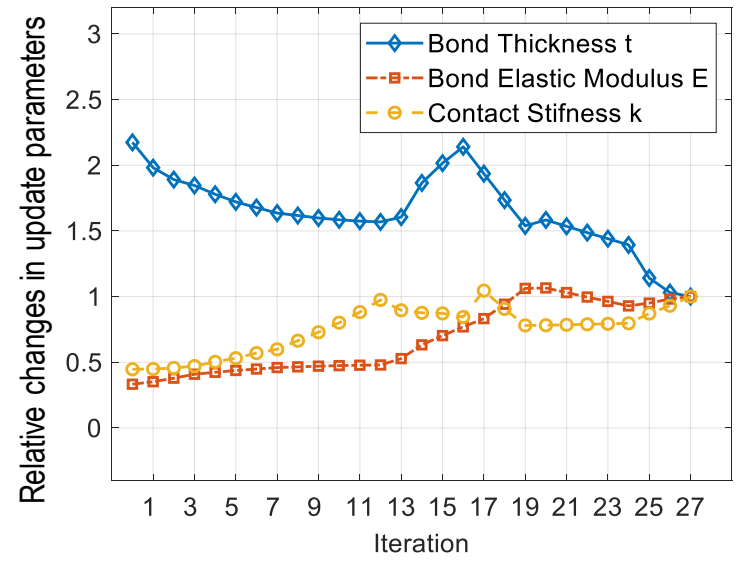

(b) Model-updating parameters

Fig. 6. Changes in model-updating frequencies and parameters

The values of the peak frequencies and model-updating parameters during the iteration process are listed in Tab. 1. The structural parameters of the FE model were identified as the numerical peak frequencies $\left(f_{1, F E M}\right.$ and $\left.f_{2, F E M}\right)$ matched with the experimental ones $\left(f_{1, \text { Exp }}\right.$ and $\left.f_{2, \text { Exp }}\right)$. After being converged, the values of the bond thickness, the bond Young's modulus, and the contact stiffness were found at $t_{b}=0.092 \mathrm{~mm}, E_{b}=9.02 \mathrm{GPa}$, $k_{z}=6.72 \mathrm{E}+12\left(\mathrm{~N} / \mathrm{m}^{2} / \mathrm{m}\right)$. The relative changes in the three model-updating parameters 
during the model-tuning process are shown in Fig. 6(b). The updated parameters at the final iteration were considered as the reference. As observed in Fig. 6(b), the relative differences were large for the earlier iterations and converged to the unity at the $27^{\text {th }}$ iteration. Particularly, the bond thickness was decreased by about $217 \%$ while the bond elastic modulus and the contact stiffness were increased by $67 \%$ and $55 \%$, respectively.

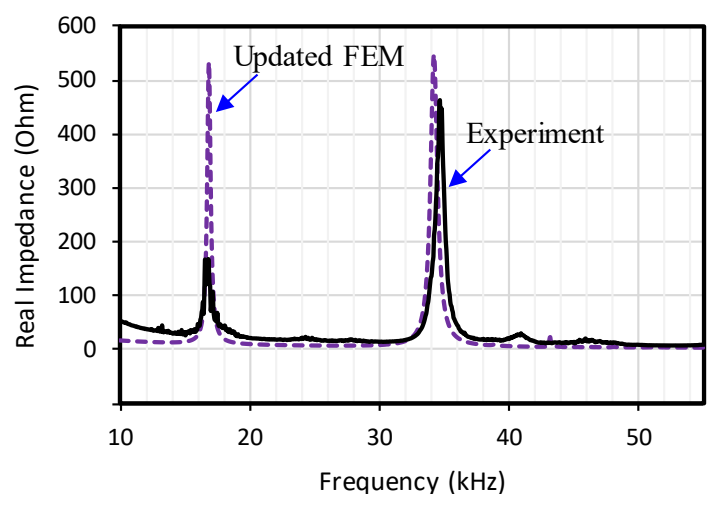

(a) Real impedance

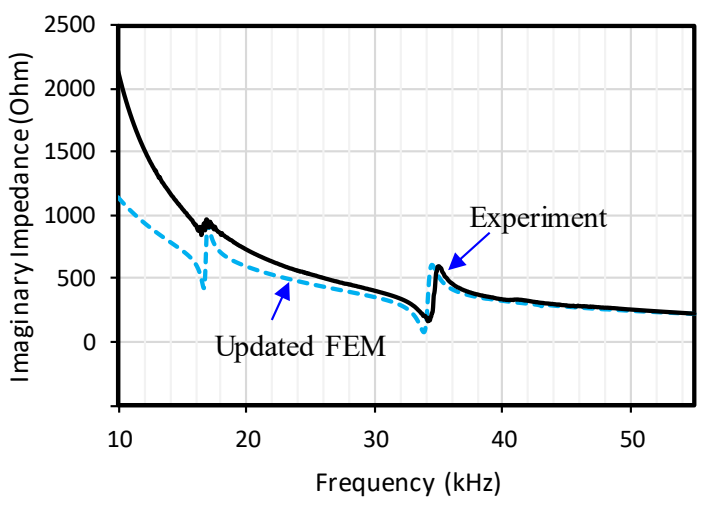

(b) Imaginary impedance

Fig. 7. EM impedance signatures: updated FE model vs experimental model

The real part of the numerical EM impedance was compared with the experimental result in Fig. 7(a). Despite certain differences in the peak magnitude, it is obvious that the peak frequencies of the FE model were consistent with those of the experimental model at the identical frequency range with similar patterns. The consistency between the two models was also observed in the imaginary part of the EM impedance, see Fig. 7(b). Conclusively, the updated FE model reproduced the experimental EM impedance signatures. It should be noted that the number of updating parameters should not much higher than the number of modal frequencies to ensure the convergence of model-updating [29,37]. In this study, only two modal frequencies are available, so three updating parameters (which are uncertain) were selected. Despite a few updating parameters, the FE model was well-identified and able to reproduce the measured impedance signatures. This evidenced the feasibility of using three updating parameters for the structural system identification.

The results showed that the structural parameters of the FE model, including the bond thickness, the bond elastic modulus, and the bolt preload-induced contact stiffness, were determined via the structural identification process. In reality, it is very challenging to measure those parameters via experiments without damaging the piezoelectric interface and the host structure. Thus, the impedance modelling strategy proposed in this study could open an alternative approach for determining the unknown structural parameters of the bolted joint in practice. Furthermore, as reported in [5] the modelupdating of contact stiffness can be used to estimate the preload force. For that, the relation between the contact stiffness and the preload force is constructed from the experiment and then used to predict the preload force from the identified contact stiffness. 


\section{SUMMARY AND CONCLUSIONS}

This study dealt with the problem of the structural parameter identification of a piezoelectric interface-bolted connection system. To address this problem, a predictive modelling strategy combining finite element (FE) modelling with a model-updating approach was proposed. At first, the basic operating principle of the piezoelectric-based smart interface was introduced. Next, a bolted girder connection was selected as a host structure to conduct real impedance measurement via the smart interface. Then, a FE model corresponding to the experiment was established by using COMSOL Multiphysics. Afterwards, the FE model was fine-tuned by a sensitivity-based model-updating algorithm, to reproduce the measured impedance responses of the connection. The highfrequency impedance signatures generated by the updated FE model were wellconsistent with the measured ones, thus evidencing the feasibility of the model for accurate impedance predictions.

By model-updating, the structural parameters of the PZT interface-bolted connection system, including the bond thickness, the bond elastic modulus, and the contact stiffness, were identified nondestructively. This study could open an alternative approach for determining the unknown structural parameters of the bonding layer in practice. Further, the model-updating of contact stiffness can help to estimate the percentage loss of the preload force. This issue remains for future investigation.

\section{ACKNOWLEDGEMENT}

This research is funded by Vietnam National Foundation for Science and Technology Development (NAFOSTED) under grant number 107.01-2019.332.

\section{REFERENCES}

[1] C. Liang, F. P. Sun, and C. A. Rogers. Coupled electro-mechanical analysis of adaptive material systems-determination of the actuator power consumption and system energy transfer. Journal of Intelligent Material Systems and Structures, 8, (4), (1997), pp. 335-343. https://doi.org/10.1177/1045389x9700800406.

[2] G. Park, H. Sohn, C. R. Farrar, and D. J. Inman. Overview of piezoelectric impedance-based health monitoring and path forward. Shock and Vibration Digest, 35, (6), (2003), pp. 451-464. https://doi.org/10.1177/05831024030356001.

[3] T.-C. Huynh, N.-L. Dang, and J.-T. Kim. Advances and challenges in impedance-based structural health monitoring. Structural Monitoring and Maintenance, 4, (4), (2017), pp. 301-329.

[4] J. Zhu, Y. Wang, and X. Qing. A real-time electromechanical impedance-based active monitoring for composite patch bonded repair structure. Composite Structures, 212, (2019), pp. 513523. https://doi.org/10.1016/j.compstruct.2019.01.035.

[5] S. Ritdumrongkul, M. Abe, Y. Fujino, and T. Miyashita. Quantitative health monitoring of bolted joints using a piezoceramic actuator-sensor. Smart Materials and Structures, 13, (1), (2003). https://doi.org/10.1117/12.482381.

[6] J. Min, S. Park, C.-B. Yun, C.-G. Lee, and C. Lee. Impedance-based structural health monitoring incorporating neural network technique for identification of damage type and severity. Engineering Structures, 39, (2012), pp. 210-220. https://doi.org/10.1016/j.engstruct.2012.01.012. 
[7] T.-C. Nguyen, T.-C. Huynh, J.-H. Yi, and J.-T. Kim. Hybrid bolt-loosening detection in wind turbine tower structures by vibration and impedance responses. Wind and Structures, 24, (4), (2017), pp. 385-403. https://doi.org/10.12989/was.2017.24.4.385.

[8] T.-C. Huynh, D.-D. Ho, N.-L. Dang, and J.-T. Kim. Sensitivity of piezoelectric-based smart interfaces to structural damage in bolted connections. Sensors, 19, (17), (2019). https://doi.org/10.3390/s19173670.

[9] K.-D. Nguyen and J.-T. Kim. Smart PZT-interface for wireless impedance-based prestressloss monitoring in tendon-anchorage connection. Smart Structures and Systems, 9, (6), (2012), pp. 489-504. https://doi.org/10.12989/sss.2012.9.6.489.

[10] C. K. Soh, K. K. H. Tseng, S. Bhalla, and A. Gupta. Performance of smart piezoceramic patches in health monitoring of a RC bridge. Smart Materials and Structures, 9, (4), (2000). https://doi.org/10.1088/0964-1726/9/4/317.

[11] J.-T. Kim, J.-H. Park, D.-S. Hong, and W.-S. Park. Hybrid health monitoring of prestressed concrete girder bridges by sequential vibration-impedance approaches. Engineering Structures, 32, (1), (2010), pp. 115-128. https://doi.org/10.1016/j.engstruct.2009.08.021.

[12] J.-T. Kim, J.-H. Park, D.-S. Hong, and D.-D. Ho. Hybrid acceleration-impedance sensor nodes on Imote2-platform for damage monitoring in steel girder connections. Smart Structures and Systems, 7, (5), (2011), pp. 393-416. https://doi.org/10.12989/sss.2011.7.5.393.

[13] R. Perera, A. Pérez, M. García-Diéguez, and J. L. Zapico-Valle. Active wireless system for structural health monitoring applications. Sensors, 17, (12), (2017). https://doi.org/10.3390/s17122880.

[14] G. Song, H. Gu, and Y.-L. Mo. Smart aggregates: multi-functional sensors for concrete structures-a tutorial and a review. Smart Materials and Structures, 17, (3), (2008). https://doi.org/10.1088/0964-1726/17/3/033001.

[15] W. Li, T. Liu, D. Zou, J. Wang, and T.-H. Yi. PZT based smart corrosion coupon using electromechanical impedance. Mechanical Systems and Signal Processing, 129, (2019), pp. 455-469. https://doi.org/10.1016/j.ymssp.2019.04.049.

[16] T.-C. Huynh, S.-Y. Lee, N.-L. Dang, and J.-T. Kim. Sensing region characteristics of smart piezoelectric interface for damage monitoring in plate-like structures. Sensors, 19, (6), (2019). https://doi.org/10.3390/s19061377.

[17] T.-C. Huynh and J.-T. Kim. Impedance-based cable force monitoring in tendon-anchorage using portable pzt-interface technique. Mathematical Problems in Engineering, 2014, (2014). https://doi.org/10.1155/2014/784731.

[18] J.-Y. Ryu, T.-C. Huynh, and J.-T. Kim. Tension force estimation in axially loaded members using wearable piezoelectric interface technique. Sensors, 19, (1), (2019). https://doi.org/10.3390/s19010047.

[19] N.-L. Dang, T.-C. Huynh, Q.-Q. Pham, S.-Y. Lee, and J.-T. Kim. Damage-sensitive impedance sensor placement on multi-strand anchorage based on local stress variation analysis. Structural Control and Health Monitoring, (2020). https://doi.org/10.1002/stc.2547.

[20] N.-L. Dang, T.-C. Huynh, and J.-T. Kim. Local strand-breakage detection in multi-strand anchorage system using an impedance-based stress monitoring method-Feasibility study. Sensors, 19, (5), (2019). https://doi.org/10.3390/s19051054.

[21] R. Tawie, H. B. Park, J. Baek, and W. S. Na. Damage detection performance of the electromechanical impedance (emi) technique with various attachment methods on glass fibre composite plates. Sensors, 19, (5), (2019). https://doi.org/10.3390/s19051000. 
[22] T.-C. Huynh and J.-T. Kim. Quantitative damage identification in tendon anchorage via PZT interface-based impedance monitoring technique. Smart Structures and Systems, 20, (2), (2017), pp. 181-195. https://doi.org/10.12989/sss.2017.20.2.181.

[23] M. Friswell and J. E. Mottershead. Finite element model updating in structural dynamics. Boston: Kluwer Academic, (1995).

[24] M. Link. Updating of analytical models-review of numerical procedures and application aspects. In Structural Dynamics Forum SD2000, Research Studies Press, Baldock, UK, (1999), pp. 193-223.

[25] B. Jaishi and W.-X. Ren. Structural finite element model updating using ambient vibration test results. Journal of Structural Engineering, 131, (4), (2005), pp. 617-628. https://doi.org/10.1061/(asce)0733-9445(2005)131:4(617).

[26] A. De Sortis and P. Paoliani. Statistical analysis and structural identification in concrete dam monitoring. Engineering structures, 29, (1), (2007), pp. 110-120. https://doi.org/10.1016/j.engstruct.2006.04.022.

[27] F. N. Catbas, S. K. Ciloglu, O. Z. H. A. N. Hasancebi, K. Grimmelsman, and A. E. Aktan. Limitations in structural identification of large constructed structures. Journal of Structural Engineering, 133, (8), (2007), pp. 1051-1066. https://doi.org/10.1061/(asce)07339445(2007)133:8(1051).

[28] R. Pasquier and I. F. C. Smith. Iterative structural identification framework for evaluation of existing structures. Engineering Structures, 106, (2016), pp. 179-194. https://doi.org/10.1016/j.engstruct.2015.09.039.

[29] T.-C. Huynh, J.-H. Park, and J.-T. Kim. Structural identification of cable-stayed bridge under back-to-back typhoons by wireless vibration monitoring. Measurement, 88, (2016), pp. 385401. https://doi.org/10.1016/j.measurement.2016.03.032.

[30] J. R. Wu and Q. S. Li. Finite element model updating for a high-rise structure based on ambient vibration measurements. Engineering Structures, 26, (7), (2004), pp. 979-990. https://doi.org/10.1016/j.engstruct.2004.03.002.

[31] D.-D. Ho, J.-T. Kim, N. Stubbs, and W.-S. Park. Prestress-force estimation in PSC girder using modal parameters and system identification. Advances in Structural Engineering, 15, (6), (2012), pp. 997-1012. https://doi.org/10.1260/1369-4332.15.6.997.

[32] M. Sanayei, B. Arya, E. M. Santini, and S. Wadia-Fascetti. Significance of modeling error in structural parameter estimation. Computer-Aided Civil and Infrastructure Engineering, 16, (1), (2001), pp. 12-27. https://doi.org/10.1111/0885-9507.00210.

[33] M. Ge and E. M. Lui. Structural damage identification using system dynamic properties. Computers $\mathcal{E}$ Structures, 83, (27), (2005), pp. 2185-2196. https://doi.org/10.1016/j.compstruc.2005.05.002.

[34] E. Aktan, N. Çatbaş, A. Türer, and Z. Zhang. Structural identification: Analytical aspects. Journal of Structural Engineering, 124, (7), (1998), pp. 817-829. https://doi.org/10.1061/(asce)0733-9445(1998)124:7(817).

[35] H. W. Shih, D. P. Thambiratnam, and T. H. T. Chan. Vibration based structural damage detection in flexural members using multi-criteria approach. Journal of Sound and Vibration, 323, (3-5), (2009), pp. 645-661. https://doi.org/10.1016/j.jsv.2009.01.019.

[36] N. Stubbs and J.-T. Kim. Damage localization in structures without baseline modal parameters. AIAA Journal, 34, (8), (1996), pp. 1644-1649. https://doi.org/10.2514/3.13284.

[37] T.-C. Huynh, S.-Y. Lee, N.-L. Dang, and J.-T. Kim. Vibration-based structural identification of caisson-foundation system via in situ measurement and simplified model. Structural Control and Health Monitoring, 26, (3), (2019). https://doi.org/10.1002/stc.2315. 
[38] G. Park, H. H. Cudney, and D. J. Inman. Feasibility of using impedance-based damage assessment for pipeline structures. Earthquake Engineering \& Structural Dynamics, 30, (10), (2001), pp. 1463-1474. https://doi.org/10.1002/eqe.72.

[39] J. Min, S. Park, and C.-B. Yun. Impedance-based structural health monitoring using neural networks for autonomous frequency range selection. Smart Materials and Structures, 19, (12), (2010). https://doi.org/10.1088/0964-1726/19/12/125011.

[40] B. Wang, L. Huo, D. Chen, W. Li, and G. Song. Impedance-based pre-stress monitoring of rock bolts using a piezoceramic-based smart washer-A feasibility study. Sensors, 17, (2), (2017). https://doi.org/10.3390/s17020250.

[41] J.-T. Kim, K.-D. Nguyen, and J.-H. Park. Wireless impedance sensor node and interface washer for damage monitoring in structural connections. Advances in Structural Engineering, 15, (6), (2012), pp. 871-885. https://doi.org/10.1260/1369-4332.15.6.871.

[42] K.-D. Nguyen, S.-Y. Lee, P.-Y. Lee, and J.-T. Kim. Wireless SHM for bolted connections via multiple PZT-interfaces and Imote2-platformed impedance sensor node. In Proceedings of the 6th International Workshop on Advanced Smart Materials and Smart Structures Technology (ANCRiSST2011), Dalian, China, (2011), pp. 25-26.

[43] J.-Y. Ryu, T.-C. Huynh, and J.-T. Kim. Experimental investigation of magnetic-mount PZTinterface for impedance-based damage detection in steel girder connection. Structural Monitoring Maintenance, 4, (3), (2017), pp. 237-253. https://doi.org/10.12989/smm.2017.4.3.237.

[44] T.-C. Huynh, N.-L. Dang, and J.-T. Kim. Preload monitoring in bolted connection using piezoelectric-based smart interface. Sensors, 18, (9), (2018). https://doi.org/10.3390/s18092766.

[45] T.-C. Huynh and J.-T. Kim. Quantification of temperature effect on impedance monitoring via PZT interface for prestressed tendon anchorage. Smart Materials and Structures, 26, (12), (2017). https://doi.org/10.1088/1361-665x/aa931b.

[46] C. W. Ong, Y. Yang, Y. T. Wong, S. Bhalla, Y. Lu, and C. K. Soh. Effects of adhesive on the electromechanical response of a piezoceramic-transducer-coupled smart system. In Smart Materials, Structures, and Systems, International Society for Optics and Photonics, Vol. 5062, (2003), pp. 241-247.

[47] M. Gresil, L. Yu, V. Giurgiutiu, and M. Sutton. Predictive modeling of electromechanical impedance spectroscopy for composite materials. Structural Health Monitoring, 11, (6), (2012), pp. 671-683. https://doi.org/10.1177/1475921712451954. 